Case Report

\title{
Chondroblastoma's Lung Metastases Treated with Denosumab in Pediatric Patient
}

\author{
Marco Focaccia, Marco Gambarotti ${ }^{2}$, Rossella Hakim ${ }^{3}$, Anna Paioli $^{3}$, Marilena Cesari $^{3}$, Benedetta Spazzoli 1 , Paolo Spinnato ${ }^{4}$, \\ Davide Donati ${ }^{1}$, Michele Rocca ${ }^{5}$, Alessandra Longhi ${ }^{3}$ \\ ${ }^{1}$ Orthopedic Division, ${ }^{2}$ Department of Pathology, ${ }^{3}$ Chemotherapy Division, ${ }^{4}$ Department of Radiology, ${ }^{5}$ General Surgery Division, IRCCS, \\ Istituto Ortopedico Rizzoli, Bologna, Italy
}

Chondroblastoma is a rare benign chondrogenic tumor that occurs in skeletally immature patients between ages 10 and 20 years old. In literature are reported few cases of lung metastases, mainly occurred after surgery or local recurrences. There is no evidence on the pathogenesis of lung metastasis, as well as pulmonary disease course. Few treatments for metastases with aggressive behavior were based on chemotherapy regimen employed in other sarcoma with no results or not satisfying ones. Denosumab is approved for treatment of giant cell tumors and it is under investigation for other giant cell-rich bone tumors. Here, we report a case of a 16-year-old male chondroblastoma of the left humerus with bilateral lung metastases at presentation and progressing during follow-up, treated with denosumab for almost 2 years. We confirm that denosumab treatment can be effective in controlling chondroblastoma metastasis and it has been a safe procedure in an adolescent patient.

Key words Chondroblastoma, Lung, Metastasis, Chemotherapy, Denosumab, Pediatrics

\section{Introduction}

Chondroblastoma is a rare benign chondrogenic tumor. It counts about $1 \%$ of all primary bone tumors. It occurs mainly in the epiphysis of long bones (distal femur, tibia, and humerus) in skeletally immature patients between ages 10 and 20 years old; males are more affected than females [1].

Histologically, it's characterized by sheets of round to ovoid cells with well-defined cytoplasm borders and grooved nucleus, eosinophilic chondroid matrix, and osteoclast like giant cells [2]. Differential diagnosis includes primarily giant cell tumor of bone. Recent genetic and immunohistochemistry studies [3] have defined a p.Lys36Met substitutions in H3F3B gene on chromosome 17 in about $98 \%$ of chondroblastomas, also resulting in the immunohistochemical expression of $\mathrm{H} 3 \mathrm{~F} 3 \mathrm{~B}$, that distinguishes it from giant cell tumor of bone which presents a H3F3A [4] mutation and from other mimickers.

Treatment is mainly surgical and based on curettage; en-bloc resection is the treatment of choice in lesions with massive bone destruction or in large recurrences. The recurrence rates are reported between 5\%-29\% after curettage and they are minimal following en-bloc resection [5]. In literature are reported few cases of lung metastases, mainly occurred after surgery or local recurrences; only three patients presented lung metastasis at diagnosis. There is no evidence on the patho- genesis of lung metastasis, as well as pulmonary disease course: some are described with aggressive behavior [6] but most cases had a benign evolution with prolonged stable disease without chemotherapy [7]. Only in a few cases, the patient deceased for lung metastases years after since diagnosis of advanced disease [8].

Few treatments for metastases with aggressive behavior were based on chemotherapy regimen employed in other sarcomas with ifosfamide, doxorubicin or vincristine, but with no results or not satisfying ones [9].

Denosumab is a human monoclonal IgG2 antibody that inhibits activation and differentiation of osteoclast-like giant cells and consequent osteolytic damage by binding RANKligand. It is approved for treatment of giant cell tumors [10] and it is under investigation for other giant cell-rich bone tumors [11,12]. A previous case report indicates a successful control of temporal bone chondroblastoma local recurrence with denosumab [13]. Here we report a case of young male chondroblastoma of the left humerus with bilateral lung metastases at presentation and progressing during follow-up, treated with denosumab for almost 2 years. 

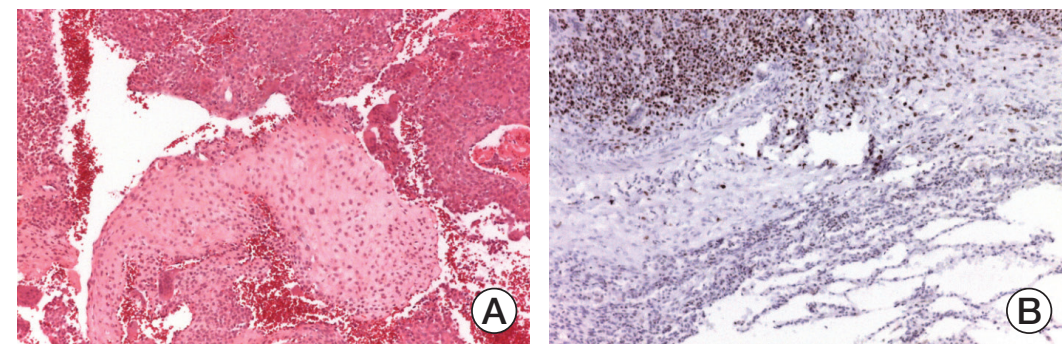

Fig. 1. (A) Humeral lesion: histological features consistent with chondroblastoma (H\&E staining, x10). (B) Lung metastasis: immunohistochemical positivity for H3F3B in neoplastic cells in the lung metastasis from chondroblastoma (x10).

\section{Case Report}

A 16-year-old male patient came to our attention complaining about left shoulder pain since October 2017, with no history of previous trauma. X-rays and magnetic resonance imaging showed a condroid osteolithic lesion at proximal left humerus, and bone scan revealed a hypercaptation in the same site. Chest computed tomography (CT) scan at diagnosis showed multiples bilateral nodules without calcification, suspicious for metastasis. A bone biopsy was performed in January 2018 at another center and histological diagnosis of chondroblastoma was performed, the specimen was reviewed and the diagnosis confirmed at our hospital. After confirmation of diagnosis, at our clinic, the patient's left humerus lesion was treated with curettage and bone cement filling. Histologically, both the biopsy and the curettage showed a proliferation of ovoid cells with an indented nucleus imparting a "coffee-bean" appearance, growing in sheets in an eosinophilic chondroid matrix, with scattered multinucleated giant cells. Neoplastic cells showed diffuse immunohistochemical positivity for H3F3B. At the postoperative follow-up, the X-rays showed surgery outcomes with no signs of local recurrence but chest CT-scan showed a progression of number and dimension of lung nodules, in particular 11 nodules in the left lung and 17 in the right lung. The positron emission tomography scan confirmed diffused bilateral lung nodules with values of standardized uptake value between 1.9 and 6.6. Surgical resection with curative aim was considered not feasible due to the diffusion and site of lung disease.

After four months of diagnosis, it was performed a diagnostic left lung metastasectomy; four subpleuric nodules ( 0.5 to $2.1 \mathrm{~cm}$ ) were removed. Wedge resection could not be performed on the right side, due to the parahiliar localization of the greater nodules. Histological examination confirmed the diagnosis of chondroblastoma pulmonary metastasis; neoplastic cells showed diffuse immunohistochemical positivity for H3F3B (Fig. 1).
At the chest computed tomografy scan two months after metastasectomy, compared to the previous, the dimensional and numerical growth of lung lesions was noticed.

Considering the progressive increase in numbers and dimensions of lung metastasis and the safety of the drug [13], we decided to treat the patient with subcutaneous 120 $\mathrm{mg}$ denosumab on a monthly basis, after the first month in which denosumab was administrated 3-times per week as for giant cell tumor protocol [10]. In consideration of the risk of osteonecrosis of jaw as side effects of the drug, reported in literature about 1\% [10], before starting denosumab therapy, the patient had X-ray orthopanthomography and oral evaluation which were normal. Calcium, phosphorus and renal function were checked before each administration, in order to exclude hypocalcemia and hypophosphatemia. A supplement of $500 \mathrm{mg}$ calcium carbonate and 400 UI vitamin D each day, was administrated during all denosumab treatment. After five months of diagnosis, chest CT scan was performed before treatment and it was repeated at every 3-month follow-ups, showing progressive minimal reduction and calcification of all residual lung nodules and presenting no new nodules until last evaluation in April 2020 after twenty months of treatment.

The treatment was well tolerated, no side effects were noticed. Every 6 months, the patient had a dentistry evaluation and yearly X-ray orthopanthomography. Serum calcium, phosphate, and creatinine were regularly checked and were in normal range. No growth arrest was reported: the patient started the treatment with a height of $164 \mathrm{~cm}$ and is now $167 \mathrm{~cm}$, in normal range for Italian growth chart. The patient was still on treatment in April 2020.

At the last follow-up, the patient was asymptomatic and the disease was still under control: the chest CT scan performed in April 2020, after 20 months since the beginning of denosumab, showed further calcification and slightly decreasing in size of pre-existing metastasis (Figs. 2 and 3) and no other nodules were detected. There were no signs of local recurrence in the left proximal humerus. 

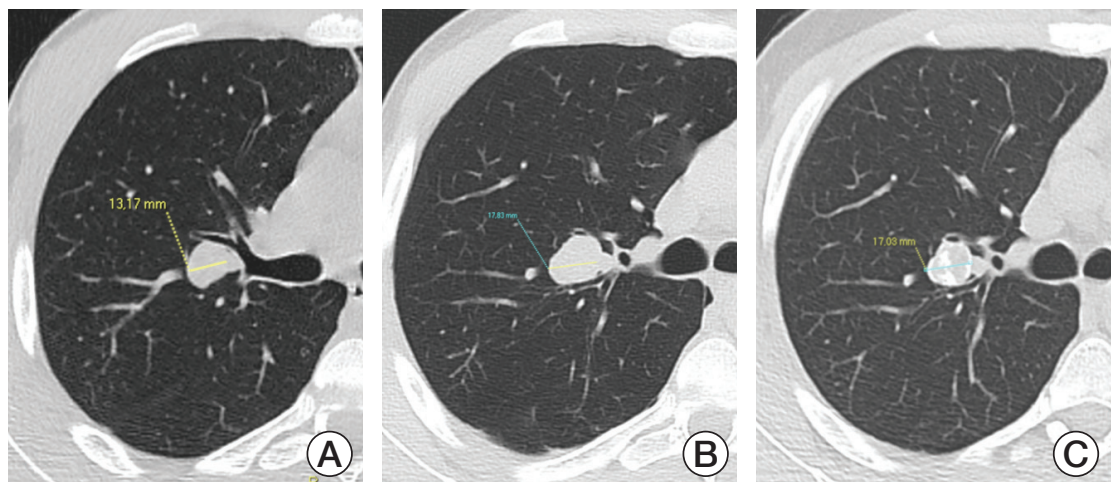

Fig. 2. Lung computed tomography, at diagnosis in January 2018 (A), at the beginning of denosumab treatment in July 2018 (B) and after 20 months of treatment in March 2020 (C), shows metastasis at the right upper lobe slightly growing in dimensions before the treatment. The metastasis dimensions remain stable after 20 months of denosumab; intralesional calcifications are detectable in the last control (C).
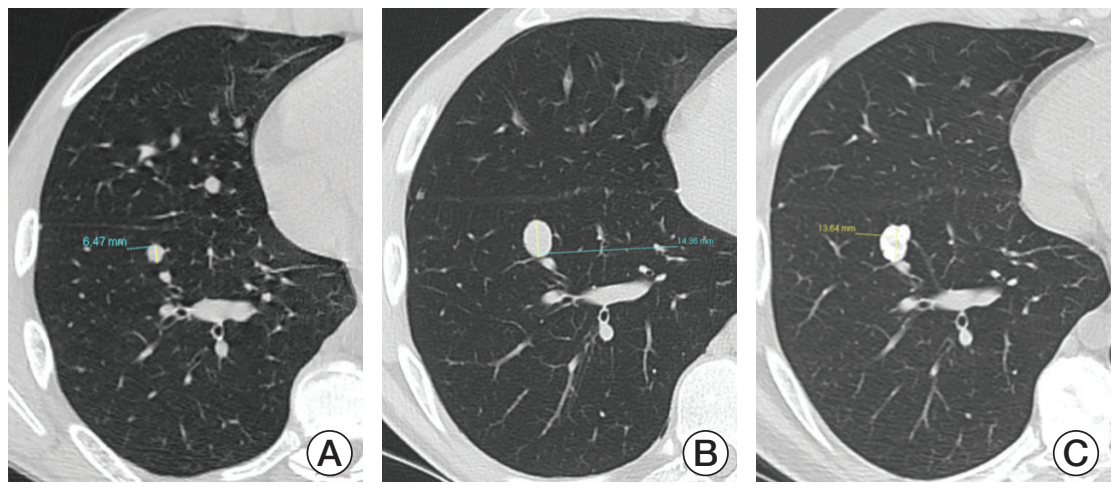

Fig. 3. Lung computed tomography, at diagnosis in January 2018 (A), at the beginning of denosumab treatment in July 2018 (B) and after 20 months of treatment in March 2020 (C), shows metastasis at the right lower lobe growing in dimensions before the treatment. The metastasis dimensions remain stable after 20 months of denosumab; intralesional calcifications are detectable in the last control (C).

\section{Discussion}

Chondroblastoma is a benign bone tumor that affects younger adults with exceptional distant metastases, more often after surgery or local recurrences. Lung metastases are rare and in literature are described only four patients, including the present case report, with onset at diagnosis (S1 Table).

Pulmonary disease is often described with a benign course but sometimes metastases had malignant behavior and patients have died because of them. In literature, there is no evidence on treatment of pulmonary disease: some authors described surgical resection, some performed lung radiotherapy and few responses were reported with conventional chemotherapy (S2 Table). Elek et al. [13] described a complete resolution of lung metastases with Cisplatin and Doxorubicin, but other authors report no responses or unsatisfying results.
Chondroblastoma shares with giant cell tumor of bone the presence of multinucleated osteoclast-like giant cells, whose treatment with denosumab is proven to be effective. Calvert and Wood [14] reported the stabilization of a temporal bone local recurrence with denosumab in an adult patient for two subsequent times. Denosumab is employed in children for other diseases like giant cell tumor, aneurismal bone cyst or osteogenesis imperfecta with good tolerance for 1-year treatment [11,12]. Chawla at al. [10] reported as more frequent long term side effects in adult patient hypophosphatemia $(5 \%)$, osteonecrosis of jaw $(3 \%)$, anemia $(2 \%)$, pain in extremity $(2 \%)$ and back pain $(1 \%)$. Serious adverse events were reported in $26 \%$ of patients and the most common was osteonecrosis of the jaw (3\%). Long-term side effects of denosumab in immature skeleton are not completely known. Only in one report [12], two out of three adolescent patients reported hypocalcemia. Another report on 20 children cancer 
patients with osteoporosis from acute linfoblastic leukemia treatment reported $40 \%$, which corresponds to eight out of 20 cases of hypocalcemia [15]. Here we report a case of a young boy with chondroblastoma of the left humerus with growing lung metastasis detected at presentation. We treated him with denosumab which proved a good tolerance and allowed the disease stabilization with lung metastases calcification as response. No local recurrence and no new nodules were noticed during almost 2 years of treatment. Due to the efficient response obtained with therapy, it was already planned to stop denosumab in April 2020 and follow the patient with chest CT scan. Stereotactic radiotherapy could be an option in case of growing nodules.

In this case report, we confirmed that denosumab treatment could be effective in controlling metastases from chondroblastoma and it has been a safe procedure in an adolescent patient for almost 2 years of therapy.

\section{Electronic Supplementary Material}

Supplementary materials are available at Cancer Research and Treatment website (https:// www.e-crt.org).

\section{Ethical Statement}

Although IRB is not requested for a single case report according to Italian rules, we have collected a written informed consent from a patient to publish his medical history and images from his radiological studies.

\section{Author Contributions}

Conceived and designed the analysis: Focaccia M, Longhi A. Collected the data: Focaccia M, Gambarotti M, Spinnato P, Longhi A. Contributed data or analysis tools: Hakim R, Paioli A, Cesari M, Spazzoli B, Spinnato P, Donati D, Rocca M.

Performed the analysis: Focaccia M, Gambarotti M, Longhi A.

Wrote the paper: Focaccia M, Gambarotti M, Longhi A.

\section{Conflicts of Interest}

Conflict of interest relevant to this article was not reported.

\section{References}

1. Dahlin DC, Ivins JC. Benign chondroblastoma: a study of 125 cases. Cancer. 1972;30:401-13.

2. John I, Inwards CY, Wenger DE, Williams DD, Fritchie KJ. Chondroblastomas presenting in adulthood: a study of 39 patients with emphasis on histological features and skeletal distribution. Histopathology. 2020;76:308-17.

3. Rehkamper J, Steinestel K, Jeiler B, Elges S, Hekeler E, Huss $\mathrm{S}$, et al. Diagnostic tools in the differential diagnosis of giant cell-rich lesions of bone at biopsy. Oncotarget. 2018;9:30106-14.

4. Righi A, Mancini I, Gambarotti M, Picci P, Gamberi G, Marraccini $C$, et al. Histone 3.3 mutations in giant cell tumor and giant cell-rich sarcomas of bone. Hum Pathol. 2017;68:128-35.

5. Chen W, DiFrancesco LM. Chondroblastoma: an update. Arch Pathol Lab Med. 2017;141:867-71.

6. Ostrowski ML, Johnson ME, Truong LD, Hicks MJ, Smith FE, Spjut HJ. Malignant chondroblastoma presenting as a recurrent pelvic tumor with DNA aneuploidy and p53 mutation as supportive evidence of malignancy. Skeletal Radiol. 1999;28:644-50.

7. Kunze E, Graewe T, Peitsch E. Histology and biology of metastatic chondroblastoma: report of a case with a review of the literature. Pathol Res Pract. 1987;182:113-23.

8. Ozkoc G, Gonlusen G, Ozalay M, Kayaselcuk F, Pourbagher A, Tandogan RN. Giant chondroblastoma of the scapula with pulmonary metastases. Skeletal Radiol. 2006;35:42-8.
9. Kyriakos M, Land VJ, Penning HL, Parker SG. Metastatic chondroblastoma: report of a fatal case with a review of the literature on atypical, aggressive, and malignant chondroblastoma. Cancer. 1985;55:1770-89.

10. Chawla S, Blay JY, Rutkowski P, Le Cesne A, Reichardt P, Gelderblom H, et al. Denosumab in patients with giant-cell tumour of bone: a multicentre, open-label, phase 2 study. Lancet Oncol. 2019;20:1719-29.

11. Raux S, Bouhamama A, Gaspar N, Brugieres L, Entz-Werle $\mathrm{N}$, Mallet $\mathrm{C}$, et al. Denosumab for treating aneurysmal bone cysts in children. Orthop Traumatol Surg Res. 2019;105:1181-5.

12. Upfill-Brown A, Bukata S, Bernthal NM, Felsenfeld AL, Nelson SD, Singh A, et al. Use of denosumab in children with osteoclast bone dysplasias: report of three cases. JBMR Plus. 2019;3:e10210.

13. Elek EM, Grimer RJ, Mangham DC, Davies AM, Carter SR, Tillman RM. Malignant chondroblastoma of the os calcis. Sarcoma. 1998;2:45-8.

14. Calvert N, Wood D. Use of denosumab in recurrent chondroblastoma of the squamous temporal bone: a case report. Clin Case Rep. 2017;5:411-3.

15. Huang TH, Liu HC, Hou JY, Chang CY, Sun FJ, Yeh TC. Efficacy and safety of denosumab therapy for low bone mineral density in childhood cancer survivors: a report of preliminary experience. Pediatr Blood Cancer. 2019;66:e27927. 